\title{
A spectral equivalent condition of the $P$-polynomial property for association schemes
}

\author{
Hirotake Kurihara \\ Department of Integrated Arts and Science \\ Kitakyushu National College of Technology \\ 5-20-1 Shii, Kokuraminamiku \\ Kitakyushu, Fukuoka, 802-0985, Japan \\ kurihara@kct.ac.jp
}

\author{
Hiroshi Nozaki \\ Department of Mathematics \\ Aichi University of Education \\ 1 Hirosawa, Igaya-cho, Kariya-city \\ Aichi, 448-8542, Japan \\ hnozaki@auecc.aichi-edu.ac.jp
}

Submitted: Feb 2, 2012; Accepted: Jun 14, 2014; Published: Jul 3, 2014

Mathematics Subject Classifications: 05E30, 05C50

\begin{abstract}
We give two equivalent conditions of the $P$-polynomial property of a symmetric association scheme. The first equivalent condition shows that the $P$-polynomial property is determined only by the first and second eigenmatrices of the symmetric association scheme. The second equivalent condition is another expression of the first using predistance polynomials.
\end{abstract}

Keywords: $P$-polynomial association scheme; distance-regular graph; graph spectrum; spectral excess theorem; predistance polynomial

\section{Introduction}

An association scheme is a finite set with higher regularity of relations among the elements of the set. As interesting objects, $P$ - or $Q$-polynomial association schemes are closely related to the theory of orthogonal polynomials or linear programming $[1,8]$, and are main topics in algebraic combinatorics. Kurihara and Nozaki [11] found a simple equivalent condition of the $Q$-polynomial property using the first and second eigenmatrices of the association scheme. In this paper, we give a "dual" version of the above theorem for the $P$-polynomial property using the first eigenmatrix whose entries are the eigenvalues of the adjacency matrices. Namely the following is a spectral equivalent condition of the $P$-polynomial property for association schemes. 
Theorem 1.1. Let $\mathfrak{X}=\left(X,\left\{R_{i}\right\}_{i=0}^{d}\right)$ be a symmetric association scheme of class $d$. Suppose that the entries $\left\{\theta_{j}\right\}_{j=0}^{d}$ of the first column of the first eigenmatrix of $\mathfrak{X}$ are mutually distinct. Then the following are equivalent:

(1) $\mathfrak{X}$ is a P-polynomial association scheme with respect to the first adjacency matrix $A_{1}$.

(2) There exists $l \in\{0,1, \ldots, d\}$ such that for each $h \in\{1,2, \ldots, d\}$,

$$
\prod_{\substack{j=1 \\ j \neq h}}^{d} \frac{\theta_{0}-\theta_{j}}{\theta_{h}-\theta_{j}}=-Q_{h}(l),
$$

where $Q_{h}(l)$ is the $(l, h)$-entry of the second eigenmatrix of $\mathfrak{X}$.

Moreover if (2) holds, then $A_{l}$ is the $d$-th matrix with respect to the resulting polynomial ordering.

After we obtained Theorem 1.1, Nomura and Terwilliger [13] gave its linear algebraic generalization. In this paper, we give a simple proof of this result based on Kurihara and Nozaki [11]. Moreover we investigate a relation between predistance polynomials and this result.

$P$-polynomial association schemes are identified with distance-regular graphs. The spectrum of a graph can give several properties of the graph. For example, we can know regularity, bipartiteness, the number of the connected components, and so on [2]. Nevertheless distance-regularity is not determined only by the spectrum of a graph. Fiol and Garriga [9] gave a characterization of distance-regularity using excesses and predistance polynomials determined by the spectra of finite graphs, and this result is called a spectral excess theorem. Here the excess of a vertex is the number of the vertices at distance $D$ from it, where $D$ is the diameter of the graph. After this work other descriptions of the spectral excess theorem were obtained by van Dam [6], and Fiol, Gago and Garriga [10].

The $P$-polynomial property demands a suitable ordering of adjacency matrices. On the other hand, Theorem 1.1 implies that the $P$-polynomial property is determined only by the spectrum of the first and the last matrices with respect to the resulting polynomial ordering. The last matrix has the information of the excess of the graph $\left(X, R_{1}\right)$. For a connected regular graph, the value corresponding to the left-hand side in (1.1) can be expressed by using the predistance polynomials. We give another expression of Theorem 1.1 by predistance polynomials of the graph $\left(X, R_{1}\right)$.

Theorem 1.2. Let $\mathfrak{X}=\left(X,\left\{R_{i}\right\}_{i=0}^{d}\right)$ be a symmetric association scheme of class $d$. Suppose that the entries $\left\{\theta_{j}\right\}_{j=0}^{d}$ of the first column of the first eigenmatrix of $\mathfrak{X}$ are mutually distinct. Let $\left\{p_{i}\right\}_{i=0}^{d}$ be the predistance polynomials of the graph $\left(X, R_{1}\right)$. Then the following are equivalent:

(1) $\mathfrak{X}$ is a P-polynomial association scheme with respect to $A_{1}$.

(2) There exists $l \in\{0,1, \ldots, d\}$ such that for each $h \in\{0,1, \ldots, d\}, p_{d}\left(\theta_{h}\right)=P_{l}(h)$ holds, where $P_{l}(h)$ is the $(h, l)$-entry of the first eigenmatrix of $\mathfrak{X}$. 


\section{Preliminaries}

\subsection{Association schemes}

We begin with a review of basic definitions concerning association schemes. The reader is referred to Bannai and Ito [1] for background materials.

A symmetric association scheme $\mathfrak{X}=\left(X,\left\{R_{i}\right\}_{i=0}^{d}\right)$ of class $d$ consists of a finite set $X$ and a set $\left\{R_{i}\right\}_{i=0}^{d}$ of binary relations on $X$ satisfying

1. $R_{0}=\{(x, x) \mid x \in X\}$,

2. $\left\{R_{i}\right\}_{i=0}^{d}$ is a partition of $X \times X$,

3. ${ }^{t} R_{i}=R_{i}$ for each $i \in\{0,1, \ldots, d\}$, where ${ }^{t} R_{i}=\left\{(y, x) \mid(x, y) \in R_{i}\right\}$,

4. the numbers $\mid\left\{z \in X \mid(x, z) \in R_{i}\right.$ and $\left.(z, y) \in R_{j}\right\} \mid$ are constant whenever $(x, y) \in$ $R_{k}$, for each $i, j, k \in\{0,1, \ldots, d\}$.

Let $k_{i}$ denote the valency of the regular graph $\left(X, R_{i}\right)$. Let $M_{X}(\mathbb{C})$ denote the algebra of matrices over the complex field $\mathbb{C}$ with rows and columns indexed by $X$. The $i$-th adjacency matrix $A_{i}$ in $M_{X}(\mathbb{C})$ of $\mathfrak{X}$ is defined by

$$
A_{i}(x, y)= \begin{cases}1 & \text { if }(x, y) \in R_{i} \\ 0 & \text { otherwise }\end{cases}
$$

The vector space $\mathfrak{A}$ spanned by $\left\{A_{i}\right\}_{i=0}^{d}$ over $\mathbb{C}$ forms a commutative algebra, and is called the Bose-Mesner algebra of $\mathfrak{X}$. It is well known that $\mathfrak{A}$ is semi-simple $[1$, Section $2.3]$, hence $\mathfrak{A}$ has the primitive idempotents $E_{0}, E_{1}, \ldots, E_{d}$. We call $m_{j}:=\operatorname{rank} E_{j}$ the multiplicities of $\mathfrak{X}$. The first eigenmatrix $P=\left(P_{i}(j)\right)_{j, i=0}^{d}$ and the second eigenmatrix $Q=\left(Q_{j}(i)\right)_{i, j=0}^{d}$ of $\mathfrak{X}$ are defined by

$$
A_{i}=\sum_{j=0}^{d} P_{i}(j) E_{j} \text { and } E_{j}=\frac{1}{|X|} \sum_{i=0}^{d} Q_{j}(i) A_{i}
$$

respectively. Note that the pair of $\left\{P_{i}(j)\right\}_{j=0}^{d}$ and $\left\{m_{j}\right\}_{j=0}^{d}$ corresponds to the spectrum of the graph $\left(X, R_{i}\right)$. In particular, we use the notation $\theta_{j}=P_{1}(j)$ for $0 \leqslant j \leqslant d$.

A symmetric association scheme is called a $P$-polynomial scheme (or a metric scheme) with respect to the ordering $\left\{A_{i}\right\}_{i=0}^{d}$ (or $\left\{R_{i}\right\}_{i=0}^{d}$ ), if for each $i \in\{0,1, \ldots, d\}$, there exists a polynomial $v_{i}$ of degree $i$, such that $A_{i}=v_{i}\left(A_{1}\right)$. Moreover, a symmetric association scheme is called a $P$-polynomial scheme with respect to $A_{1}$ (or $R_{1}$ ) if the symmetric association scheme has the $P$-polynomial property with respect to some ordering $A_{0}, A_{1}, A_{\xi_{2}}, A_{\xi_{3}}, \ldots, A_{\xi_{d}}$. Dually, a symmetric association scheme is called a $Q$ polynomial scheme (or a cometric scheme) with respect to the ordering $\left\{E_{j}\right\}_{j=0}^{d}$, if for each $j \in\{0,1, \ldots, d\}$, there exists a polynomial $v_{j}^{*}$ of degree $j$, such that $|X| E_{j}=v_{j}^{*}\left(|X| E_{1}\right)$, where the multiplication is the entrywise product. 
Remark 2.1. For a $P$-polynomial scheme $\left(X,\left\{R_{i}\right\}_{i=0}^{d}\right)$ with respect to $R_{1}$, the graph $\left(X, R_{1}\right)$ is distance-regular. Conversely, for a distance-regular graph $(X, R)$ with diameter $d$, letting $R_{i}$ be the collection $(x, y) \in X \times X$ such that the distance between $x$ and $y$ is $i$ for $i \in\{0,1, \ldots, d\}$, we have a $P$-polynomial scheme $\left(X,\left\{R_{i}\right\}_{i=0}^{d}\right)$ with respect to the ordering $\left\{R_{i}\right\}_{i=0}^{d}[1]$.

Remark 2.2. Kurihara and Nozaki [11] found a simple equivalent condition of the $Q$ polynomial property using the character table of the association scheme. The detail of the equivalent condition is as follows. For a symmetric association scheme $\mathfrak{X}=\left(X,\left\{R_{i}\right\}_{i=0}^{d}\right)$ such that $\left\{Q_{1}(i)\right\}_{i=0}^{d}$ are mutually distinct, $\mathfrak{X}$ is a $Q$-polynomial association scheme with respect to $E_{1}$ if and only if there exists $l \in\{0,1, \ldots, d\}$ such that for each $h \in\{1,2, \ldots, d\}$,

$$
\prod_{\substack{i=1 \\ i \neq h}}^{d} \frac{Q_{1}(0)-Q_{1}(i)}{Q_{1}(h)-Q_{1}(i)}=-P_{h}(l) .
$$

\subsection{The predistance polynomials of graphs}

As we see in Remark 2.1, a distance-regular graph $\Gamma$ has the structure of a $P$-polynomial scheme and so has polynomials $\left\{v_{i}\right\}$. In this section, we give "predistance polynomials" for any regular connected graph, and we consider relations between predistance polynomials and distance-regular graphs.

All graphs in this paper are assumed to be finite. Let $\Gamma=(X, R)$ be a connected regular graph with the adjacency matrix $A$ and the spectrum $\left\{\theta_{0}^{m_{0}}, \theta_{1}^{m_{1}}, \ldots, \theta_{d}^{m_{d}}\right\}$, where $\theta_{0}>\theta_{1}>\cdots>\theta_{d}$. Let $Z(t):=\prod_{j=0}^{d}\left(t-\theta_{j}\right) \in \mathbb{R}[t]$. We define the inner product on $\mathbb{R}[t] /(Z)$ by

$$
\langle p, q\rangle=\frac{1}{|X|} \sum_{j=0}^{d} m_{j} p\left(\theta_{j}\right) q\left(\theta_{j}\right)
$$

for $p, q \in \mathbb{R}[t] /(Z)$. The predistance polynomials $p_{0}, p_{1}, \ldots, p_{d}$ of $\Gamma$ are the unique polynomials satisfying $\operatorname{deg} p_{i}=i$ and for $i, j \in\{0,1, \ldots, d\},\left\langle p_{i}, p_{j}\right\rangle$ is $p_{i}\left(\theta_{0}\right)$ if $i=j$ and 0 otherwise. It is well known that $p_{i}\left(\theta_{0}\right)>0$ for any $i \in\{0,1, \ldots, d\}[6]$.

For a graph of diameter $D$, the excess $\gamma_{x}$ of a vertex $x$ is the number of the vertices at distance $D$ from $x$. An application of predistance polynomials and excesses is shown in the following theorem. Namely, distance-regularity is determined by predistance polynomials and excesses.

Theorem 2.3 (Excess theorem (Fiol, Gago and Garriga [10])). Let $(X, R)$ be a connected regular graph with $d+1$ distinct eigenvalues and diameter $D=d,\left\{p_{i}\right\}_{i=0}^{d}$ be the predistance polynomials, and $\gamma_{x}$ be the excess of $x \in X$. Then

$$
\frac{1}{|X|} \sum_{x \in X} \gamma_{x} \leqslant p_{d}\left(\theta_{0}\right)
$$

Moreover equality is attained if and only if $(X, R)$ is a distance-regular graph. 
There are many researches of distance-regularity or walk-regularity using predistance polynomials $[3,4,5,6,7,9,12]$.

Remark 2.4. If $\Gamma$ is a distance-regular graph, the predistance polynomials $\left\{p_{i}\right\}$ of $\Gamma$ coincide with the polynomial $\left\{v_{i}\right\}$ associated with the $P$-polynomial structure of $\Gamma[6]$.

The following lemma is used later.

Lemma 2.5. Let $\Gamma$ be a connected regular graph with the spectrum $\left\{\theta_{0}^{m_{0}}, \theta_{1}^{m_{1}}, \ldots, \theta_{d}^{m_{d}}\right\}$ and the predistance polynomials $\left\{p_{i}\right\}_{i=0}^{d}$. Then for each $h \in\{1,2, \ldots, d\}$,

$$
\prod_{\substack{j=1 \\ j \neq h}}^{d} \frac{\theta_{0}-\theta_{j}}{\theta_{h}-\theta_{j}}=-\frac{m_{h} p_{d}\left(\theta_{h}\right)}{p_{d}\left(\theta_{0}\right)}
$$

Proof. Put $f_{h}(t)=\prod_{j=1, j \neq h}^{d}\left(t-\theta_{j}\right)$, for $i \in\{1,2, \ldots, d\}$. These polynomials have degree $d-1$ which is expressed as a linear combination of $\left\{p_{i}\right\}_{i=0}^{d-1}$, hence they are orthogonal to $p_{d}$. Thus, note that $\Gamma$ is connected and $m_{0}=1$, and we have

$$
0=|X|\left\langle p_{d}, f_{h}\right\rangle=\sum_{i=0}^{d} m_{i} p_{d}\left(\theta_{i}\right) f_{h}\left(\theta_{i}\right)=p_{d}\left(\theta_{0}\right) f_{h}\left(\theta_{0}\right)+m_{h} p_{d}\left(\theta_{h}\right) f_{h}\left(\theta_{h}\right) .
$$

Therefore we obtain the desired equation.

\section{Proof of Theorem 1.1}

First, we prove that (1) implies (2) in Theorem 1.1. Suppose $\mathfrak{X}$ is a $P$-polynomial association scheme with respect to $A_{1}$. Let $\left\{A_{i}\right\}_{i=0}^{d}$ be the $P$-polynomial ordering and $\left\{v_{i}\right\}_{i=0}^{d}$ be the polynomials associated with the $P$-polynomial structure. For each $h \in\{1,2 \ldots, d\}$, we put $\kappa_{h}=\prod_{j=1, j \neq h}^{d}\left(\theta_{0}-\theta_{j}\right) /\left(\theta_{h}-\theta_{j}\right)$, and define the matrix

$$
M_{h}^{*}:=\prod_{\substack{j=1 \\ j \neq h}}^{d} \frac{A_{1}-\theta_{j} I}{\theta_{h}-\theta_{j}}
$$

in $\mathfrak{A}$. Since $M_{h}^{*}$ is a polynomial in $A_{1}$ of degree $d-1$, it can be expressed as a combination of $\left\{A_{i}\right\}_{i=0}^{d-1}$. Thus we have

$$
M_{h}^{*} \circ A_{d}=0
$$

where $\circ$ denotes the Hadamard product, that is, the entry-wise matrix product. On the other hand, we consider the expansion of $M_{h}^{*}$ in terms of the primitive idempotents $\left\{E_{j}\right\}_{j=0}^{d}$. Since $A_{1} E_{j}=\theta_{j} E_{j}$, we have

$$
M_{h}^{*} E_{j}= \begin{cases}\kappa_{h} E_{0} & \text { if } j=0 \\ E_{h} & \text { if } j=h \\ 0 & \text { otherwise. }\end{cases}
$$


This means $M_{h}^{*}=\kappa_{h} E_{0}+E_{h}$. Hence, by (3.1), it follows that $E_{h} \circ A_{d}=-\kappa_{h} A_{d} /|X|$, that is, $Q_{h}(d)=-\kappa_{h}$. Therefore the desired result follows.

Conversely, we prove that (2) implies (1) in Theorem 1.1. The following lemmas are used later.

Lemma 3.1 ([11]). For mutually distinct $\beta_{1}, \beta_{2}, \ldots, \beta_{d}$, the following formal identity holds:

$$
\sum_{i=1}^{d} \beta_{i}^{k} \prod_{\substack{j=1 \\ j \neq i}}^{d} \frac{x-\beta_{j}}{\beta_{i}-\beta_{j}}=x^{k}
$$

for all $k \in\{0,1, \ldots, d-1\}$.

We say that $A_{j}$ appears in an element $M$ of $\mathfrak{A}$ if there exists a non-zero complex number $\alpha$ such that $M \circ A_{j}=\alpha A_{j}$. Let $N_{k}^{*}$ denote the set of indices $j$ such that $A_{j}$ appears in $A_{1}^{k}$ but does not appear in $A_{1}^{l}$ for each $0 \leqslant l \leqslant k-1$. We remark that $N_{0}^{*}=\{0\}$ and $N_{1}^{*}=\{1\}$. Moreover, if $\theta_{0}$ is distinct from $\left\{\theta_{j}\right\}_{j=1}^{d}$, then $J=|X| \prod_{j=1}^{d} \frac{A_{1}-\theta_{j} I}{\theta_{0}-\theta_{j}}$ holds by the property of the Hoffman polynomial. Therefore $\{0,1, \ldots, d\}=\bigcup_{k=0}^{d} N_{k}^{*}$ (a disjoint union).

Lemma 3.2. Suppose $\mathfrak{X}$ is a symmetric association scheme of class $d$ satisfying $\theta_{0}$ is distinct from $\left\{\theta_{i}\right\}_{i=1}^{d}$. Then, the following are equivalent.

(1) $\mathfrak{X}$ is a P-polynomial scheme with respect to $A_{1}$.

(2) $\bigcup_{k=0}^{d-1} N_{k}^{*} \neq\{0,1, \ldots, d\}$.

Proof. Suppose $\mathfrak{X}$ is a $P$-polynomial scheme with respect to $A_{1}$. Let $\left\{A_{i}\right\}_{i=0}^{d}$ be the $P$ polynomial ordering. Then there exist polynomials $v_{i}$ of degree $i$, such that $v_{i}\left(A_{1}\right)=A_{i}$ for any $i$. This implies that $N_{i}^{*}=\{i\}$ for any $i$, that is, $\bigcup_{k=0}^{d-1} N_{k}^{*}=\{0,1, \ldots, d-1\}$.

Suppose $\bigcup_{k=0}^{d-1} N_{k}^{*} \neq\{0,1, \ldots, d\}$ holds. Since $\bigcup_{k=0}^{d} N_{k}^{*}=\{0,1, \ldots, d\}$, it follows $N_{d}^{*}$ is not empty. If $N_{i}^{*}$ is empty for some $i$, then $N_{i+1}^{*}$ is also empty. This means that if $N_{d}^{*}$ is not empty, then $N_{i}^{*}$ is not empty for any $i$. Since $N_{0}^{*}, N_{1}^{*}, \ldots, N_{d}^{*}$ are disjoint, $N_{i}^{*}$ has size 1 for each $i \in\{0,1, \ldots, d\}$. Put $N_{i}^{*}=\left\{\xi_{i}\right\}$ and order $A_{\xi_{0}}, A_{\xi_{1}}, \ldots, A_{\xi_{d}}$. Then we can construct polynomials $v_{i}$ of degree $i$ such that $v_{i}\left(A_{1}\right)=A_{\xi_{i}}$, and the first statement follows.

Remark 3.3. From the proof of Lemma 3.2, an ordering of a $P$-polynomial association scheme with respect to $A_{1}$ is uniquely determined.

Let us return to prove that (2) implies (1) in Theorem 1.1. We have

$$
A_{1}^{k}=\frac{\theta_{0}^{k}}{|X|} J+\sum_{h=1}^{d} \theta_{h}^{k} E_{h} .
$$


By our assumption, it follows that

$$
E_{h} \circ A_{l}=\frac{Q_{h}(l)}{|X|} A_{l}=-\frac{1}{|X|} \prod_{\substack{j=1 \\ j \neq h}}^{d} \frac{\theta_{0}-\theta_{j}}{\theta_{h}-\theta_{j}} A_{l} .
$$

By Lemma 3.1,

$$
A_{1}^{k} \circ A_{l}=\left(\frac{\theta_{0}^{k}}{|X|} J+\sum_{h=1}^{d} \theta_{h}^{k} E_{h}\right) \circ A_{l}=\frac{1}{|X|}\left(\theta_{0}^{k}-\sum_{h=1}^{d} \theta_{h}^{k} \prod_{\substack{j=1 \\ j \neq h}}^{d} \frac{\theta_{0}-\theta_{j}}{\theta_{h}-\theta_{j}}\right) A_{l}=0
$$

for every $k \leqslant d-1$. This means that $l$ is not an element of $N_{k}^{*}$ for every $k \leqslant d-1$. By Lemma 3.2, there exists an ordering $\left\{A_{\xi_{i}}\right\}_{i=0}^{d}$ such that $\mathfrak{X}$ is a $P$-polynomial scheme with respect to $\left\{A_{\xi_{i}}\right\}_{i=0}^{d}$. Moreover we have $\xi_{d}=l$. Therefore the desired result follows.

\section{Proof of Theorem 1.2}

First, we prove that (1) implies (2) in Theorem 1.2. Suppose $\mathfrak{X}$ is a $P$-polynomial association scheme with respect to $A_{1}$. Let $\left\{v_{i}\right\}_{i=0}^{d}$ be the polynomials associated with the $P$ polynomial structure of $\mathfrak{X}$. Then $\left\{v_{i}\right\}_{i=0}^{d}$ coincide with the predistance polynomials $\left\{p_{i}\right\}_{i=0}^{d}$ of the graph $\left(X, R_{1}\right)$ by Remarks 2.1 and 2.4. Therefore we have $p_{d}\left(\theta_{h}\right)=v_{d}\left(\theta_{h}\right)=P_{d}(h)$ for each $h \in\{0,1, \ldots, d\}$.

Next, we prove that (2) implies (1) in Theorem 1.2. Suppose $p_{d}\left(\theta_{h}\right)=P_{l}(h)$ holds for each $h \in\{0,1, \ldots, d\}$. Then by Lemma 2.5 and $k_{l} Q_{h}(l)=m_{h} P_{l}(h)[1$, Theorem 3.5, page $62]$ we have

$$
\prod_{\substack{j=1 \\ j \neq h}}^{d} \frac{\theta_{0}-\theta_{j}}{\theta_{h}-\theta_{j}}=-\frac{m_{h} p_{d}\left(\theta_{h}\right)}{p_{d}\left(\theta_{0}\right)}=-\frac{m_{h} P_{l}(h)}{k_{l}}=-Q_{h}(l)
$$

for each $h \in\{1,2, \ldots, d\}$. By Theorem $1.1, \mathfrak{X}$ is a $P$-polynomial association scheme with respect to $A_{1}$.

\section{Acknowledgments}

The authors thank Edwin van Dam for pointing out that the value in Theorem 1.1 can be expressed by predistance polynomials, and the referees for some useful suggestions that improved the presentation. The authors are also grateful to Kazumasa Nomura, Hajime Tanaka and Paul Terwilliger for careful reading and insightful comments. The second author was supported by JSPS KAKENHI Grant Number 25800011.

\section{References}

[1] E. Bannai and T. Ito. Algebraic combinatorics. I. The Benjamin/Cummings Publishing Co. Inc., Menlo Park, CA, 1984. 
[2] D. Cvetković, P. Rowlinson and S. Simić. Eigenspaces of graphs. Encyclopedia of Mathematics and its Applications vol. 66, Cambridge University Press, Cambridge, 1997.

[3] C. Dalfó, E.R. van Dam, M.A. Fiol and E. Garriga. Dual concepts of almost distanceregularity and the spectral excess theorem. Discrete Math., 312(17):2730-2734, 2012.

[4] C. Dalfó, E.R. van Dam, M.A. Fiol, E. Garriga and B.L. Gorissen. On almost distance-regular graphs. J. Combin. Theory Ser. A, 118(3):1094-1113, 2011.

[5] C. Dalfó, M.A. Fiol and E. Garriga. Characterizing $(\ell, m)$-walk-regular graphs. Linear Algebra Appl., 433(11-12):1821-1826, 2010.

[6] E.R. van Dam. The spectral excess theorem for distance-regular graphs: a global (over)view. Electron. J. Combin., 15(1): Research Paper 129, 10, 2008.

[7] E.R. van Dam and M.A. Fiol. A short proof of the odd-girth theorem. Electron. J. Combin., 19(3): Research Paper 12, 5, 2012.

[8] P. Delsarte. An algebraic approach to the association schemes of coding theory. Philips Res. Rep. Suppl., (10):vi+97, 1973.

[9] M.A. Fiol and E. Garriga. From local adjacency polynomials to locally pseudodistance-regular graphs. J. Combin. Theory Ser. B, 71(2):162-183, 1997.

[10] M.A. Fiol, S. Gago and E. Garriga A simple proof of the spectral excess theorem for distance-regular graphs. Linear Algebra Appl., 432(9): 2418-2422, 2010.

[11] H. Kurihara and H. Nozaki. A characterization of $Q$-polynomial association schemes. J. Combin. Theory Ser. A, 119(1): 57-62, 2012.

[12] W. Liu and J. Lin. k-walk-regular digraphs. J. Math. Res. Expo., 31(4): 637-642, 2011.

[13] K. Nomura and P. Terwilliger. Tridiagonal matrices with nonnegative entries. Linear Algebra Appl., 434: 2527-2538, 2011. 